\title{
Cardiovascular gene expression profiles of dioxin exposure in zebrafish embryos
}

\author{
Heather M. Handley-Goldstone ${ }^{1}$, Matthew W. Grow ${ }^{2,3}$, John J. Stegeman ${ }^{1}$ \\ ${ }^{1}$ Woods Hole Oceanographic Institution, Biology Department, Woods Hole MA 02543 \\ ${ }^{2}$ Indiana University School of Medicine, Center for Medical Genomics, Indianapolis IN 46202 \\ ${ }^{3}$ Massachusetts General Hospital, Cardiovascular Research Center, Charlestown MA
}

Short Title: dioxin-modulated gene expression in zebrafish

\section{Correspondence:}

Heather M. Handley-Goldstone

Redfield 3-52, MS\#32

Woods Hole Oceanographic Institution

Woods Hole MA 02543

Phone: (508) 289-3472

Fax: (508) 457-2134

E-mail: hhandley@alum.mit.edu

\section{Supplementary Data:}

Handley-Goldstone_GenePix_data.xls

Handley-Goldstone_Resolver_results.xls 


\begin{abstract}
2,3,7,8-Tetrachlorodibenzo-p-dioxin (TCDD) is a widespread environmental contaminant that causes altered heart morphology, circulatory impairment, edema, hemorrhage, and early life stage mortality in fish. TCDD toxicity is largely dependent upon the aryl hydrocarbon receptor, but understanding of the molecular mechanism of cardiovascular embryotoxicity remains incomplete. In order to identify genes potentially involved in cardiovascular impacts, we constructed custom cDNA microarrays consisting of 4,896 zebrafish adult heart cDNA clones and over 200 genes with known developmental, toxicological, and housekeeping roles. Gene expression profiles were obtained for 3-day old zebrafish following early embryonic exposure to either 0.5 or $5.0 \mathrm{nM}$ TCDD. 516 clones were significantly differentially expressed (p-value $<$ 0.005) under at least one treatment condition; 123 high-priority clones were selected for further investigation. CYP1A, CYP1B1, and other members of the AHR gene battery, were strongly and dose-dependently induced by TCDD. Importantly, altered expression of cardiac sarcomere components, including cardiac troponin T2 and multiple myosin isoforms, was consistent with the hypothesis that TCDD causes dilated cardiomyopathy. Observed increases in expression levels of mitochondrial energy transfer genes also may be related to cardiomyopathy. Other TCDD-responsive genes included fatty acid and steroid metabolism enzymes, ribosomal and signal transduction proteins, and 18 ESTs with no known protein homologs. As the first broadscale study of TCDD-modulated gene expression in a non-mammalian system, this work provides an important perspective on mechanisms of TCDD toxicity.
\end{abstract}




\section{Introduction}

2,3,7,8-Tetrachlorodibenzo- $p$-dioxin (TCDD) is a widespread environmental contaminant and a potent disruptor of cardiovascular development in vertebrates. Hallmarks of TCDD embryotoxicity in fish include reduced heart size, circulatory failure, pericardial and yolk sac edema, hemorrhage, and early life stage mortality (Belair et al., 2001; Henry et al., 1997; Hornung et al., 1999). In zebrafish, weakened cardiac contraction can be observed shortly after hatching, followed by congestion and reduced perfusion of peripheral vascular beds, and finally, edema and hemorrhage (Belair et al., 2001; Dong et al., 2002; Henry et al., 1997; Teraoka et al., 2002). This progression is generally conserved across fish species (Hornung et al., 1999; Wisk and Cooper, 1990), and is reminiscent of congestive heart failure.

In avian embryos, TCDD causes dilated cardiomyopathy by inhibiting cardiomyocyte proliferation during a period of significant ventricular muscle growth and rearrangement (Ivnitski et al., 2001; Sedmera et al., 2000). Reduced cardiac efficiency leads to congestive heart failure with manifest edema and hemorrhage (Ivnitski et al., 2001; Walker and Catron, 2000). Cardiac remodeling processes in fish are variable and poorly understood, but cross-species similarity in the overt toxicity of TCDD implies a common molecular mechanism.

TCDD toxicity is largely dependent on the aryl hydrocarbon receptor (AHR) (FernandezSalguero et al., 1996; Lin et al., 2002; Peters et al., 1999; Staples et al., 1998; Teraoka et al., 2003), a ligand-activated transcription factor with strong affinity for dioxins and related planar halogenated aromatic hydrocarbons. Binding of TCDD by cytosolic AHR causes activation, nuclear translocation, and dimerization with aryl hydrocarbon receptor nuclear translocator (ARNT). The AHR-ARNT complex acts via DNA sequence motifs, known variously as AHR-, dioxin-, or xenobiotic-response elements (AHRE, DRE or XRE), to modulate gene expression. Transcriptional regulation is thought to be the primary means by which AHR mediates dioxin toxicity, but the identity of toxicity-causing target genes remains uncertain.

CYP1A induction is the most sensitive known response to AHR activation and may contribute to dioxin toxicity. The timing, distribution, and dose-dependence of CYP1A expression are all strongly correlated with symptoms of embryotoxicity in fish (Cantrell et al., 1998; Cantrell et al., 1996; Gannon et al., 2000; Guiney et al., 1997; Toomey et al., 2001). CYP1A could cause toxicity via aberrant reactive oxygen production in the presence of substrates that are tightly bound but poorly metabolized (Schlezinger et al., 2000; Schlezinger et 
al., 1999). The protective effects of antioxidants indicate that reactive oxygen species (ROS) are involved in TCDD embryotoxicity (Cantrell et al., 1996). However, direct assessments of the role of CYP1A in embryotoxicity have yielded conflicting results (Carney et al., 2004; Teraoka et al., 2003).

AHR is capable of regulating expression of numerous other genes that could play important roles in embryotoxicity. Microarray-based gene expression profiling and serial analysis of gene expression (SAGE) have provided a list of several hundred TCDD-responsive candidate genes in mammals. Most of this work has focused on liver tissue and cultured hepatocytes (Frueh et al., 2001; Kurachi et al., 2002; Puga et al., 2000; Zeytun et al., 2002), but spleen and thymus tissues (Zeytun et al., 2002), vascular smooth muscle cells (Guo et al., 2004; Karyala et al., 2004) and lung epithelial cells (Martinez et al., 2002) have also been interrogated. While some general trends are emerging from these studies, substantial disparities emphasize the importance of dose, tissue-type, and likely numerous other biological factors in shaping molecular responses to TCDD. Thus, it is difficult to gauge the relevance of available data to the specific problem of cardiovascular embryotoxicity.

The goal of the current work was to use cDNA microarrays to identify TCDD-responsive genes involved in developmental toxicity. Expression profiling of 3-day old zebrafish following early embryonic exposure to two doses of TCDD revealed changes consistent with cardiomyopathy, including suppression of cardiac troponin T2 and induction of cardiac myosin isoforms. Other changes in gene expression suggest subtle modulations in basal cellular functions, including mitochondrial energy transfer, fatty acid and steroid metabolism, signal transduction, and ribosomal machinery.

\section{Methods}

\section{Chemicals}

\section{2,3,7,8-tetrachloro[6- $\left.{ }^{3} \mathrm{H}\right]$ dibenzo- $p$-dioxin (ChemSyn Laboratories) 2,3,7,8-} tetrachlorodibenzo-p-dioxin (ChemService) and were obtained from providers in toluene solutions. ${ }^{3} \mathrm{H}-\mathrm{TCDD}$ alone or a 1:1 mixture of tritiated and unlabeled TCDD was dried and dissolved in DMSO at least 24 hrs prior to use. 


\section{Experimental Design}

Zebrafish embryos (strain TL) were exposed to 0.05\% (vol/vol) DMSO or appropriate TCDD/DMSO solution in E3 egg water $\left(5 \mathrm{mM} \mathrm{NaCl}, 0.17 \mathrm{mM} \mathrm{KCl}, 0.33 \mathrm{mM} \mathrm{CaCl}_{2}\right.$, and 0.33 $\mathrm{mM} \mathrm{MgSO}_{4}$ )for $1.5 \mathrm{hrs}$ beginning at approximately the 1000-cell stage. Embryos were then rinsed thoroughly and maintained in clean E3 egg water at $28^{\circ} \mathrm{C}$. Approximately $24 \mathrm{hrs}$ after dosing, triplicate samples of 3 embryos per treatment group were dissolved in Solvable reagent (Packard). Samples were mixed with Scintiverse II scintillation fluid (Packard) and darkadapted prior to liquid scintillation counting. At 72 hours post fertilization (hpf), 50-100 larvae per treatment group were collected for RNA preparation. Samples were either flash-frozen and stored at $-80^{\circ} \mathrm{C}$ or transferred to RNALater (Ambion) and stored at $-20^{\circ} \mathrm{C}$.

Samples for microarray analysis were obtained from two separate experiments. In the 0.5 nM TCDD experiment, control and treatment groups of $\sim 100$ embryos each were drawn from a single pool of embryos from multiple mating pairs. Triplicate hybridizations were performed using three distinct DMSO-TCDD sample pairs (i.e. biological replication only). In the $5.0 \mathrm{nM}$ TCDD experiment, treatment groups of $\sim 100$ embryos were taken from individual matings; 400 embryos pooled from the same clutches comprised a control group. Six hybridizations with cDNA from four treatment groups provided both biological and technical replication. Due to differences in sampling design, data from the two experiments were not subjected to direct statistical comparisons.

The sampling design for morphological and RT-PCR dose-response assessments was similar to that for the low-dose microarray experiment. Replicate experiments were performed using distinct pools of embryos that were split into treatment groups of 100-200 embryos each. cDNA Microarrays

A cDNA library made from zebrafish adult heart tissue was a kind gift of Dr. Ashok Srinivasan. cDNA probes were generated by PCR amplification from bacterial lysates or plasmid DNA preparations using standard SP6, T3, and T7 primers. Amplification success was monitored by gel electrophoresis of 12 reactions per 96-well plate. PCR products were filter purified (Millipore Multiscreen-96 PCR Purification Plates), dried by vacuum centrifugation, and reconstituted in $25 \mu 13 x \mathrm{SSC}+0.1 \%$ Sarkosyl. cDNA probes were arrayed onto Corning CMTGAPS slides using a GMS 417 Arrayer (Genetic Microsystems) equipped with four standard pins set to $250 \mu \mathrm{m}$ center-to-center feature spacing. Printed cDNAs were immobilized by UV 
cross-linking (Stratalinker® UV Crosslinker, Stratagene). Arrays were washed 2 min each in $95^{\circ} \mathrm{C}$ nuclease-free water and 95\% ethanol, air-dried, and stored in a light-protected dessication chamber. Array quality was assessed using homotypic hybridizations, performed according to the protocol below with aliquots of a single adult zebrafish heart RNA preparation.

\section{Microarray Hybridization}

Total RNA was isolated from whole embryos using TriZol reagent (Invitrogen). Amino-allyl modified cDNA was generated by reverse transcription in the presence of amino-allyl-dUTP. Total RNA (15-25 $\mu \mathrm{g})$ spiked with A. thaliana chloroplast mRNA (100, 250, and $500 \mathrm{ng}$ of Cab, $R C A$, and $r b c L$ RNA, respectively; SpotReport ${ }^{\circledR}-3$ Array Validation Kit, Stratagene) was incubated with $5 \mu \mathrm{g}$ oligo-dT ${ }_{(20)} \mathrm{N}$ anchored primer for $10 \mathrm{~min}$ at $65^{\circ} \mathrm{C}$, then chilled on wet ice. 5x First-strand buffer $(4 \mu 1)$ and $0.1 \mathrm{mM}$ DTT $(2 \mu 1)$ were added for a final reaction volume of 20 $\mu 1$. Remaining reagents were then added for final reaction conditions of $1 \mathrm{x}$ first-strand synthesis buffer, 10mM DTT, 0.5mM each dATP, dCTP and dGTP, 0.3mM dTTP, 0.2mM amino-allyldUTP, and 1000U Superscript II reverse transcriptase. Reverse transcription reactions were run $2.5 \mathrm{hrs}$ at $42^{\circ} \mathrm{C}$, then inactivated by buffering with $0.5 \mathrm{M}$ EDTA and incubating $5 \mathrm{~min}$ at $95^{\circ} \mathrm{C}$.

RNA was eliminated by alkaline hydrolysis in $0.2 \mathrm{~N} \mathrm{NaOH}$ and RNase digestion (Ambion RNase cocktail). cDNA purification and buffer exchange was accomplished by filterpurification according to standard protocols (QiaQuick PCR Purification Kit, Qiagen), except that Qiagen buffers PE and EB were replaced by 75\% ethanol and distilled water, respectively. cDNAs were dried by vacuum centrifugation and stored at $-20^{\circ} \mathrm{C}$.

For CyDye post-labeling, cDNAs were redissolved in $10 \mu \mathrm{l} 0.1 \mathrm{M} \mathrm{NaHCO}_{3}(\mathrm{pH} 9.0)$ containing amine-reactive Cy3 or Cy5 (Amersham Biosciences), then incubated 1.5-2 hrs at room temperature in darkness. The labeling reaction was quenched by $15 \mathrm{~min}$ incubation with excess hydroxylamine. Following addition of $35 \mu \mathrm{l} 100 \mathrm{mM} \mathrm{NaOAc}(\mathrm{pH} 5.2)$ and $50 \mu \mathrm{l}$ nuclease-free water, labeled cDNA was purified using QiaQuick PCR Purification columns (Qiagen) according to standard protocols. cDNA concentrations were determined spectrophotometrically $\left(\mathrm{A}_{260}, \mathrm{~A}_{280}\right)$, then equal quantities of paired Cy3- and Cy5-labeled cDNAs were combined and dried by vacuum centrifugation.

Immediately prior to hybridization, labeled target cDNA was dissolved in 3x SSC with $0.1 \%$ SDS, $0.4 \mu \mathrm{g} / \mu 1$ poly-A blocker (Sigma), $0.4 \mu \mathrm{g} / \mu$ l yeast tRNA (Invitrogen), and $0.8 \mu \mathrm{g} / \mu \mathrm{l}$ sonicated salmon sperm DNA (Fisher Scientific), then denatured $2 \mathrm{~min}$ at $95^{\circ} \mathrm{C}$. Overnight 
hybridizations were performed at $65^{\circ} \mathrm{C}$ in sealed hybridization chambers containing a reservoir of 2x SSC. Hybridized arrays were washed 3-5 min each with 2x SSC + 0.1\% SDS, 1x SSC, and $0.1 x$ SSC, then dipped briefly into distilled water and isopropanol, and air-dried.

Microarray Data Analysis

Array image capture and analysis was performed using an Axon 4000A scanner (10 $\mu \mathrm{m}$ resolution) equipped with GenePix 3.0 software. GenePix results files were deposited into the Rosetta Resolver Enterprise Gene Expression Data Analysis System (Rosetta Biosoftware, Kirkland, WA). Rosetta Resolver generates background-subtracted ratio data by applying a platform-specific error model to mean fluorescence intensities and standard deviations calculated by GenePix. Intra-array normalization is performed according to a piece-wise linear function that fits and corrects for intensity non-linearity; this method is a modification of that proposed by Schadt and colleagues (Schadt et al., 2002). Data from replicate hybridizations are combined to obtain error-weighted mean ratio data for each treatment group; the contribution of individual data points to the mean is determined by the calculated measurement error. Features with $\log$ (intensity) $<-2.0$ were excluded from analyses, and features with $p$-value $<0.005$ for the group mean ratio were considered differentially expressed.

\section{Real-time RT-PCR}

Total RNA was prepared using TriZol reagent (Invitrogen), then treated with DNase I (Ambion DNA-free ${ }^{\mathrm{TM}}$ ). cDNA was generated by oligo-dT-primed reverse transcription of $2 \mu \mathrm{g}$ total RNA with Superscript II RT (Invitrogen). Reactions containing all materials except reverse transcriptase ( $\mathrm{RT}^{-}$controls) served as controls for genomic DNA contamination. Real-time PCR reactions consisting of 1x SYBR ${ }^{\circledR}$ Green PCR Master Mix (Applied Biosystems), $1 \mu 1 \mathrm{cDNA}$ (or $\mathrm{RT}^{-}$control reactions), and $400 \mathrm{nM}$ each primer, were subjected to instrument default cycling parameters, including dissociation curve analysis (ABI Prism 7000). Threshold-crossing cycle numbers $(\mathrm{Ct})$ were determined at background-subtracted fluorescence $\mathrm{r}=0.2$, and relative expression ratios $(\mathrm{R})$ were calculated according to the previously described (Pfaffl, 2001) equation:

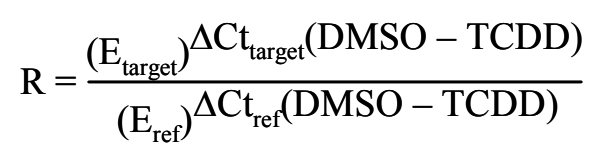


where $\beta$-actin was the reference gene, and amplification efficiencies (E) were determined as the slope of the log-linear regression of fluorescence data from the exponential phase of amplification (LinRegPCR, Ramakers et al., 2003). Mean E values were calculated for each technical replicate and used to calculate ratios for each sample in that group. $\mathrm{RT}^{-}$control reactions did not produce significant amplification signal within the range of cycle numbers used for relative quantitation, and thus, were not included in calculations of relative expression levels.

\section{Results}

\section{Cardiovascular cDNA microarrays}

Zebrafish cardiovascular cDNA microarrays consisted of 5,184 cDNA probes (see supplemental data for complete probe list and microarray data) immobilized on glass slides. The vast majority of probes $(4,896)$ were amplified from arbitrarily selected, uncharacterized adult heart cDNA clones. The originating adult heart cDNA library was comprised of approximately $65 \%$ unique clones and 35\% redundant copies of high abundance transcripts (based on sequencing of 200 randomly selected clones, data not shown). Thus, the arrayed probes likely represent approximately 2,800 unique genes. These were supplemented with probes for genes of known developmental (e.g. Nkx2-5, GATA family members) or toxicological (e.g. AHR, CYP1A) relevance, constantly expressed housekeeping genes (e.g. $\beta$-actin), and exogenous hybridization controls (SpotReport ${ }^{\mathrm{TM}}-3$ cDNA Array Validation System, Stratagene).

Homotypic control hybridizations using total RNA from adult heart tissue revealed consistent feature morphology, strong signal:noise ratios, and minimal systematic bias. In a typical example, the mean feature diameter was $190.88 \pm 23.36 \mu \mathrm{m}$ and features with abnormal (i.e. nonround) morphology were rare. The median signal:noise ratios for Cy3 and Cy5 channels were 12:1 and 9:1, respectively. The best-fit line for the fluorescence intensity scatter plot had an unadjusted slope of 1.19 (Figure $1, \mathrm{R}^{2}=0.91$ ) and the $99.7 \%$ confidence interval ( 3 standard deviations from the population mean) of median-centered data fell at a Cy3:Сy5 ratio of \pm 1.78 . Experimental design

Gene expression profiles were obtained from $72 \mathrm{hpf}$ embryos exposed to either $0.5 \mathrm{nM}$ TCDD (1.84 $\pm 0.42 \mathrm{ng}$ TCDD/g embryo, $\mathrm{n}=3$ treatment groups) or $5.0 \mathrm{nM}$ TCDD (10.74 \pm 1.38 ng TCDD/g embryo, $\mathrm{n}=4$ treatment groups) at the 1000-cell stage. In the $0.5 \mathrm{nM}$ TCDD group, there was a slight increase in the occurrence of mild pericardial edema and most embryos 
showed modest reductions in the rate of blood flow in the tail (Figure 2). In contrast, exposure to 5.0 nM TCDD produced maximal responses, including severe edema and severe to complete circulatory failure in $100 \%$ of assayed embryos. However, neither dose caused a significant increase in mortality through $96 \mathrm{hpf}$. These two concentrations represented the extremes of the dose-response curves, which were similar to those observed in other zebrafish strains (Henry et al., 1997).

All microarray control features demonstrated the expected responses (or lack thereof) to TCDD exposure (Table 1). Arabidopsis thaliana mRNAs spiked into experimental samples at equimolar concentrations showed no significant deviations from 1:1 ratios. Known housekeeping genes (i.e. $\beta$-actin and ARNT2) also were unaffected by TCDD exposure. In contrast, CYP1A was strongly and dose-dependently induced by TCDD. AHR2 showed a subtle but highly significant induction similar to that previously observed (Andreasen et al., 2002).

Real-time RT-PCR measurements of control genes were generally consistent with microarray results. Prior to normalization, RT-PCR data indicated no significant change in expression of ARNT2, but showed a significant trend toward down-regulation of $\beta$-actin (Figure 3a, singlefactor ANOVA p-value <0.05). A similar downward trend was seen in many of the genes assayed, including those expected to be up-regulated, suggesting that this trend was artifactual. Thus, RT-PCR data were normalized to $\beta$-actin. Both $\beta$-actin-normalized and raw RT-PCR data indicated strong induction of CYP1A at 0.5-15 nM TCDD (Figure 3b, single-factor ANOVA pvalue $<0.005)$. However, the magnitude of induction varied markedly between biological replicates and did not follow the expected dose-response curve (Figure 3b).

\section{TCDD-modulated gene expression}

516 clones were significantly differentially expressed ( $p$-value $<0.005$ ) at one or both TCDD concentrations. Overall, changes in expression levels were relatively subtle, with the median magnitude of change falling between 1.30 and 1.35 for both doses. Less than $10 \%$ of differentially expressed clones showed significant changes at both doses, and 131 clones were differentially expressed only at the lower dose. The total number of differentially expressed clones was greater at 5.0 nM TCDD (385 vs. 182), but this may be attributable, in part, to differences in statistical power. High-dose mean data were based on 4 biological and 2 technical replicates, while 3 biological replicates were used in the low-dose experiment. Analyses based on any subset of three high-dose biological replicates resulted in numbers of significant changes 
similar to those observed for the low dose. Thus, direct statistical comparisons between doses were avoided so as to not introduce sampling artifacts.

At this level of statistical confidence, the expected false discovery rate was 52 clones, approximately $10 \%$ of all detected changes. To limit the number of false positives passed to further investigation, 123 high-priority clones were selected using three additional criteria:

1) P-value $<0.005$ at BOTH doses (51 clones),

2) P-value $<0.005$ AND absolute fold change $\geq 1.8$ (99.7\% C.I.) at either dose (65 clones), or

3) Concordant evidence from redundant probes (12 genes, 2 ESTs), as determined from firstpass sequencing of approximately 300 clones with p-values $<0.005$. These redundant clones comprised nearly one third of all clones sequenced.

Ultimately, high-quality sequence data was obtained for 119 of 123 high-priority clones. Translated BLAST searches against public databases identified 42 known genes (Table 2) and 18 ESTs with no known homologs (Table 3). Known genes were classified into five broad functional groups, discussed below.

This subset of genes/ESTs reflected general trends seen in the larger dataset. The number of high-priority differentially expressed genes was slightly greater at $5.0 \mathrm{nM}$ TCDD (56 vs. 49 genes/ESTs), but the average magnitude of change was not significantly different (2.54 \pm 0.87 vs. 2.76 \pm 1.09 ). Up-regulated genes/ESTs far outnumbered those down-regulated; only 11 genes, 4 ESTs, and 4 unidentified clones were suppressed by TCDD.

\section{Metabolic genes}

TCDD exposure resulted in differential expression of genes involved in multiple metabolic pathways, most notably xenobiotic metabolism (Table 2a). CYP1A, CYP1B, and glutathione S transferase $\pi$, all members of the mammalian AHR gene battery, were dose-dependently induced. Major vault protein, a membrane channel involved in multi-drug resistance, was also induced. Pyrimidine 5' nucleotidase and the steroid biosynthesis enzyme $20 \beta$-hydroxysteroid dehydrogenase also were dose-dependently induced.

Effects on fatty acid regulators were mixed. Acyl-coA dehydrogenase and TP- $\beta$ showed signs of subtle induction by TCDD. In contrast, expression of liver-basic fatty acid binding protein (LB-FABP) was dose-dependently suppressed. 


\section{Cardiovascular genes}

Genes encoding components of cardiac muscle sarcomeres showed mixed responses to TCDD exposure (Table 2b). Cardiac $\alpha$-actin showed signs of slight suppression, but this effect could not be confirmed by RT-PCR (data not shown). In contrast, expression of several cardiac myosin isoforms, not all of which could be definitively identified, appeared to be induced. In both cases, these effects were most pronounced at $0.5 \mathrm{nM}$ TCDD. However, as previously noted, direct statistical comparison between doses was not performed because of possible differences in statistical power.

Cardiac troponin T2 (tnnt2) probes provided conflicting data. Multiple clones indicated significant induction of tnnt 2 at both dose levels, while a single clone suggested dose-dependent suppression of tnnt 2 expression. Real-time RT-PCR confirmed the latter result, indicating $\leq 1.8$ fold suppression of cardiac troponin T2 (Figure 4a, single-factor ANOVA p-value $<0.01$ ). To address the possibility of cross-hybridization artifacts, expression of three additional troponin isoforms was assayed by RT-PCR. Skeletal slow muscle troponin T showed a trend toward slight suppression (Figure 4a, single-factor ANOVA p-value >0.05), while skeletal fast muscle troponin $\mathrm{T}$ was significantly induced (Figure 4a, single-factor ANOVA p-value $<0.01$ ). Skeletal troponin $\mathrm{C}$ levels were unaffected (not shown).

\section{Mitochondrial genes}

Highly redundant probe sets suggested subtle induction of several protein-coding mitochondrial genes, including cytochrome b (cytb), cytochrome c oxidase (CCO), and NADH dehydrogenase (ND) (Table 2c). We attempted to verify suggested alterations in cytb, CCO subunit I, and ND subunit 2 expression using real-time RT-PCR. Results from one biological replicate were consistent with 1.2-1.5-fold induction of all three genes by TCDD, but the second biological replicate showed no significant increase in expression levels. Overall, variability between replicate samples obscured any statistical significance (Figure 4b, ANOVA p-value > $0.05)$.

Three cDNA clones matched ATP synthase sequences in public databases. One was identified as mitochondrial ATP synthase 6 by alignment with the zebrafish mitochondrial genome sequence. This probe indicated slight upregulation, similar to other mitochondrial genes. RT-PCR measurements failed to confirm any significant induction of mitochondrial ATP synthase 6 (single-factor ANOVA p-value $>0.05$, data not shown). Limited sequence data 
precluded definitive identification of the remaining two clones, one of which indicated suppression, the other slight induction.

Ribosomal genes

Genes encoding both RNA and protein components of the ribosomal machinery tended to be down-regulated by TCDD exposure (Table 2d). RT-PCR failed to detect significant differences in rRNA expression levels (not shown). RT-PCR verification of changes in levels of transcripts encoding ribosomal proteins was not attempted.

\section{Miscellaneous}

Eight additional TCDD-responsive genes either could not be classified or were the sole representative of a functional category (Table 2e). Two calcium-transporting ATPases were upregulated by TCDD. Small membrane protein 1, protein kinase C inhibitor protein 1, annexin XIa, and cryptochrome 1a were all significantly induced by both concentrations of TCDD. $\beta$ spectrin and chaperonin containing t-complex protein 1 were both induced by $0.5 \mathrm{nM}$ TCDD.

\section{Discussion}

We have generated cDNA microarrays representing approximately 2,800 zebrafish genes. We used these arrays to identify 516 cDNAs that were differentially expressed in 3 day old zebrafish following early embryonic exposure to approximate cardiovascular ED10 and ED100 concentrations of TCDD. A subset of high-priority cDNAs chosen for further investigation consisted of 42 known genes and 18 ESTs.

Dose level was a primary factor determining transcriptional responses to TCDD exposure. Less then ten percent of all differentially expressed clones met statistical significance criteria at both doses, and many genes were differentially expressed only at the lower dose. Likewise, Martinez and colleagues (Martinez et al., 2002) found that more than half of all TCDD-regulated genes were differentially expressed at only one of three doses. Genes that are responsive to TCDD over a large range of exposure levels may be of interest as biomarkers; certainly, this has been the case for CYP1A. The remaining genes highlight the diversity of TCDD dose-response relationships and the importance of considering low-dose adaptive responses as distinct from toxicity-related changes apparent at higher doses.

Only two genes - CYP1A and CYP1B1 - were induced $>4$-fold. CYP1A induction is a universal, sensitive response to TCDD exposure in vertebrates, and CYP1B1 induction by TCDD 
has been observed in several systems (Tang et al., 1996; Walker et al., 1999). However, these genes are not expressed in normal zebrafish embryos (Andreasen et al., 2002; Yamazaki et al., 2002), suggesting that ectopic induction of genes that are normally "turned off" may be a more important mechanism of action for TCDD than is modulation of normal cardiovascular programs. Such effects would be largely missed using the current microarray platform, as it is built from cDNAs expressed in normal adult heart tissue.

Subtle modulation of constitutively expressed genes is interesting in itself. Widespread, subtle changes in gene expression, as opposed to strong pressure on specific pathways, have been noted in other TCDD gene expression profiling experiments (Frueh et al., 2001; Puga et al., 2000). Signal compression does not completely explain this trend, since microarray measurements of CYP1A and AHR2 induction were similar to both current and previously published RT-PCR data (Andreasen et al., 2002). Additionally, Martinez and coworkers (Martinez et al., 2002) confirmed the magnitude of several subtle TCDD-induced changes detected by microarray. Thus, gene expression profiling is reaffirming the pleiotropic nature of impacts by TCDD, as well as the unique sensitivity of the CYP1 family to regulation by TCDD.

While the biological importance of low-magnitude changes in gene expression remains an open question, there is growing recognition that subtle modulation of multiple members of a single pathway can be physiologically relevant. This idea has driven the recent development of microarray analysis techniques, such as Gene-Set Enrichment Analysis (Mootha et al., 2003), designed to detect changes that are not statistically significant at the level of a single gene but become significant when functional groups are considered as an entity. A posteriori identification of differentially expressed cDNAs makes the current data unsuitable for such analyses. However, sarcomeric components and mitochondrial energy transfer genes seem to be compelling examples of over-represented functional groups.

The current data reflect whole-organism average responses that could result from more dramatic effects in specific tissues. For example, the heart makes up less than $2 \%$ of the total body mass of a 3-day old zebrafish. A ten-fold change in cardiac expression of a gene that is highly expressed throughout the embryo would produce less than a $20 \%$ change in overall expression level. Unfortunately, high background expression precludes reliable detection of tissue-specific changes using in situ methods, and larval zebrafish organs are prohibitively small for individual analysis. 


\section{TCDD-induced cardiomyopathy}

Observed differential expression of sarcomeric genes lends weight to the idea that developing fish suffer the same cardiac pathology as TCDD-exposed chicken embryos, namely dilated cardiomyopathy. Increased myosin expression is apparent in both cases (Walker and Catron, 2000). The significance of this elevation is uncertain, but the observation is not unprecendented; induction of sarcomeric genes, including troponins and myosins, has been documented in human cardiomyopathies (Barrans et al., 2002; Haase et al., 2002; Hwang et al., 2002). Observed reductions in troponin T2 transcript levels are consistent with dilated myopathy, and could be causally related to toxicity, just as loss-of-function mutations in human homologs are the leading causes of congenital cardiomyopathies (Seidman and Seidman, 2001). Thus, the current results are in accord with data from congenital and TCDD-induced cardiomyopathy in other species.

Discrepancies between microarray and RT-PCR data for cardiac tnnt2 do not interfere with this conclusion. The disparity may be explained either by cross-hybridization of microarray probes with other troponin $\mathrm{T}$ isoforms or by sequence-specific dye incorporation artifacts that are relatively common in microarray experiments without dye swapping. While all cDNA probes derived from the same region of the tnnt2 gene, slight differences in the 5' extent of the cDNA may have contributed to different hybridization affinities or dye bias. Control hybridizations had indicated minimal dye bias, so limited sample RNA was conserved by eliminating dye swapping. RT-PCR, which is not subject to such sequence-dependent artifacts, provides robust evidence for differential expression of cardiac and skeletal muscle troponin $\mathrm{T}$ isoforms.

Reduced cardiac size in TCDD-exposed fish seems contradictory to cardiomyopathy, but may be explained by differences in hemodynamic forces. Dilated cardiomyopathy typically results in increased ventricular diameter due to distension of the weakened muscle wall by fluid pressure. In the case of fish, TCDD-induced congestion of peripheral vasculature and red blood cell loss may relieve such pressure. The only obvious distending force is pericardial edema, which elongates the heart. Thus, the disruption of cardiomyocyte proliferation that leads to dilated cardiomyopathy in chicken could produce an atypical myopathy with reduced cardiac size in fish.

A pressing question is the nature of the relationship between sarcomeric gene expression and cardiomyocyte proliferation or cardiomyopathy. Uncoordinated expression of sarcomeric components could lead to sarcomere misassembly and weakened cardiac muscle. Alternatively, 
reduced troponin T levels could simply reflect a reduced number of troponin-expressing cells. It is impossible to distinguish such possibilities with the data currently available.

Direct regulation of sarcomeric genes by AHR may be possible. In silico searches suggest potential AHR binding sites upstream of mammalian myosins, tropomyosins, and inhibitory troponin (Fielden and Zacharewski, 2000), as well as zebrafish cardiac troponin T2 (HandleyGoldstone, unpublished). However, no functional data are available for these motifs. It will be important to determine which factors regulate sarcomeric gene expression and what role(s) they play in toxicity.

\section{Mitochondrial gene expression and reactive oxygen}

The current data suggest modest induction of mitochondrial electron transport genes. The failure of real-time RT-PCR to confirm the statistical significance of microarray results may be due to differences in experimental design. Only two biological replicates were used for RTPCR, as compared to at least three in all microarray experiments. Furthermore, the RT-PCR replicates showed obvious differences in response levels, with replicate \#2 consistently demonstrating smaller magnitudes of change. This was true for CYP1A and cardiac troponin T2, but the disparity did not impact the statistical significance of these larger changes to the same degree. TCDD uptake was not monitored in RT-PCR experiments; variation in uptake efficiency may explain both the variability between biological replicates and the early plateau in doseresponse curves.

In contrast, changes in levels of mitochondrial rRNAs indicated by microarray analyses may represent false positives. Detection of rRNAs was unexpected, as these transcripts are not polyadenylated and cDNA synthesis for both microarray hybridization and PCR was oligo-dT primed. In this case, RT-PCR failed to demonstrate even a trend consistent with the microarray data. However, no RT-PCR data are available for ribosomal protein-encoding transcripts and this remains an interesting question relating to TCDD effects on mitochondrial function.

Elevated levels of mitochondrial electron transport proteins could contribute to toxicity via reactive oxygen-mediated processes. Both CYP1A(1) (Schlezinger et al., 2000; Schlezinger et al., 1999) and mitochondria have been identified as AHR-dependent sources of ROS (Senft et al., 2002a; Senft et al., 2002b). Increased respiration-dependent reactive oxygen production by mitochondria has been observed in TCDD-treated mouse liver (Senft et al., 2002a; Senft et al., 2002b). In cardiac mitochondria, TCDD-induced ROS production has been linked to decoupling 
of respiration and downstream oxidative phosphorylation processes (Nohl et al., 1989). TCDDinduced differential expression of mitochondrial and downstream energy transfer genes has been observed in this and other studies (Frueh et al., 2001; Kurachi et al., 2002), and suggests a mechanism for decoupling of mitochondrial electron transfer associated with ROS production.

Mitochondrial ROS production might, in turn, be one pathway to cardiac toxicity. Induction of mitochondrial electron transfer proteins, including NADH dehydrogenase, has been seen in human cardiomyopathies (Hwang et al., 2002). Evidence of a causal link between excess ROS production and cardiac pathologies is increasing (Dhalla et al., 2000; Ide et al., 1999; Sorescu and Griendling, 2002).

\section{Conclusions}

As the first characterization of TCDD gene expression profiles in a non-mammalian system, this work provides a unique perspective and lends weight to emerging trends, particularly the plieotropic and dose-specific nature of transcriptional modulation by TCDD. Furthermore, observed derangements in cardiac and mitochondrial gene expression provide evidence for cardiomyopathy in zebrafish and bring an enhanced degree of unity to ongoing studies of cardiovascular toxicity in fish and birds.

Not surprisingly, though, this work has generated more intriguing questions than conclusive answers. What, if any, are the physiological impacts of widespread, subtle changes in gene expression? What roles do changes in sarcomeric and mitochondrial gene expression play in toxicity? Perhaps most exciting of all, what can the 18 TCDD-responsive ESTs identified here reveal about previously unknown aspects of TCDD toxicity?

\section{Acknowledgements}

Special thanks to the faculty and staff of the Massachusetts General Hospital Cardiovascular Research Center, particularly Dr. Mark Fishman and Dr. Ashok Srinivasan. Thanks to the staff of the Harvard Center for Genomics Research for technical assistance in microarray production and use, and to Rosetta Biosoftware for analysis tools and support. This work was supported by funding from the National Institutes of Health. 


\section{Supplementary Data}

Results from Rosetta Resolver analyses are included in the MS Excel workbook file Handley-Goldstone_Resolver_results.xls. Raw microarray data from GenePix results files are included in the Microsoft Excel workbook file Handley-Goldstone_GenePix_data.xls.

Hybridization images may be obtained upon request.

\section{References}

Andreasen, E. A., J. M. Spitsbergen, R. L. Tanguay, J. J. Stegeman, W. Heideman and R. E. Peterson (2002). Tissue-specific expression of AHR2, ARNT2, and CYP1A in zebrafish embryos and larvae: effects of developmental stage and 2,3,7,8-tetrachlorodibenzo-pdioxin exposure. Toxicol Sci 68(2): 403-19.

Barrans, J. D., P. D. Allen, D. Stamatiou, V. J. Dzau and C. C. Liew (2002). Global gene expression profiling of end-stage dilated cardiomyopathy using a human cardiovascularbased cDNA microarray. Am J Pathol 160(6): 2035-43.

Belair, C. D., R. E. Peterson and W. Heideman (2001). Disruption of erythropoiesis by dioxin in the zebrafish. Dev Dyn 222(4): 581-94.

Cantrell, S. M., J. Joy-Schlezinger, J. J. Stegeman, D. E. Tillitt and M. Hannink (1998). Correlation of 2,3,7,8-tetrachlorodibenzo-p-dioxin-induced apoptotic cell death in the embryonic vasculature with embryotoxicity. Toxicol Appl Pharmacol 148(1): 24-34.

Cantrell, S. M., L. H. Lutz, D. E. Tillitt and M. Hannink (1996). Embryotoxicity of 2,3,7,8tetrachlorodibenzo-p-dioxin (TCDD): the embryonic vasculature is a physiological target for TCDD-induced DNA damage and apoptotic cell death in Medaka (Orizias latipes). Toxicol Appl Pharmacol 141(1): 23-34.

Carney, S. A., R. E. Peterson and W. Heideman (2004). 2,3,7,8-Tetrachlorodibenzo-p-dioxin activation of the aryl hydrocarbon receptor/aryl hydrocarbon receptor nuclear translocator pathway causes developmental toxicity through a CYP1A-independent mechanism in zebrafish. Mol Pharmacol 66(3): 512-21.

Dhalla, N. S., R. M. Temsah and T. Netticadan (2000). Role of oxidative stress in cardiovascular disease. J Hypertens 18(6): 655-673.

Dong, W., H. Teraoka, K. Yamazaki, S. Tsukiyama, S. Imani, T. Imagawa, J. J. Stegeman, R. E. Peterson and T. Hiraga (2002). 2,3,7,8-tetrachlorodibenzo-p-dioxin toxicity in the zebrafish embryo: local circulation failure in the dorsal midbrain is associated with increased apoptosis. Toxicol Sci 69(1): 191-201. 
Fernandez-Salguero, P. M., D. M. Hilbert, S. Rudikoff, J. M. Ward and F. J. Gonzalez (1996). Aryl-hydrocarbon receptor-deficient mice are resistant to 2,3,7,8-tetrachlorodibenzo-pdioxin-induced toxicity. Toxicol Appl Pharmacol 140(1): 173-9.

Fielden, M. R. and T. R. Zacharewski (2000). Toxicology in silico: in search of target genes for the nuclear aryl hydrocarbon receptor complex. Keystone Symposia: Molecular Toxicology, Toxicogenomics and Bioinformatics Applied to Drug Discovery.

Frueh, F. W., K. C. Hayashibara, P. O. Brown and J. P. Whitlock, Jr. (2001). Use of cDNA microarrays to analyze dioxin-induced changes in human liver gene expression. Toxicol Lett 122(3): 189-203.

Gannon, M., D. Gilday and A. B. Rifkind (2000). TCDD induces CYP1A4 and CYP1A5 in chick liver and kidney and only CYP1A4, an enzyme lacking arachidonic acid epoxygenase activity, in myocardium and vascular endothelium. Toxicol Appl Pharmacol 164(1): 24-37.

Guiney, P. D., R. M. Smolowitz, R. E. Peterson and J. J. Stegeman (1997). Correlation of 2,3,7,8-tetrachlorodibenzo-p-dioxin induction of cytochrome P4501A in vascular endothelium with toxicity in early life stages of lake trout. Toxicol Appl Pharmacol 143(2): 256-73.

Guo, J., M. Sartor, S. Karyala, M. Medvedovic, S. Kann, A. Puga, P. Ryan and C. R. Tomlinson (2004). Expression of genes in the TGF-beta signaling pathway is significantly deregulated in smooth muscle cells from aorta of aryl hydrocarbon receptor knockout mice. Toxicol Appl Pharmacol 194(1): 79-89.

Haase, D., M. H. Lehmann, M. M. Korner, R. Korfer, H. H. Sigusch and H. R. Figulla (2002). Identification and validation of selective upregulation of ventricular myosin light chain type 2 mRNA in idiopathic dilated cardiomyopathy. Eur J Heart Fail 4(1): 23-31.

Henry, T. R., J. M. Spitsbergen, M. W. Hornung, C. C. Abnet and R. E. Peterson (1997). Early life stage toxicity of 2,3,7,8-tetrachlorodibenzo-p-dioxin in zebrafish (Danio rerio). Toxicol Appl Pharmacol 142(1): 56-68.

Hornung, M. W., J. M. Spitzbergen and R. E. Peterson (1999). 2,3,7,8-Tetrachlorodibenzo-pdioxin alters cardiovascular and craniofacial development and function in sac fry of rainbow trout (Oncorhynchus mykiss). Toxicological Sciences 47(1): 40-51.

Hwang, J. J., P. D. Allen, G. C. Tseng, C. W. Lam, L. Fananapazir, V. J. Dzau and C. C. Liew (2002). Microarray gene expression profiles in dilated and hypertrophic cardiomyopathic end-stage heart failure. Physiol Genomics 10(1): 31-44.

Ide, T., H. Tsutsui, S. Kinugawa, H. Utsumi, D. Kang, N. Hattori, K. Uchida, K. Arimura, K. Egashira and A. Takeshita (1999). Mitochondrial electron transport complex I is a potential source of oxygen free radicals in the failing myocardium. Circ Res 85: 357-363. 
Ivnitski, I., R. Elmaoued and M. K. Walker (2001). 2,3,7,8-Tetrachlorodibenzo-p-dioxin (TCDD) Inhibition of Coronary Development is Preceded by a Decrease in Myocyte Proliferation and an Increase in Cardiac Apoptosis. Teratol 64: 201-212.

Karyala, S., J. Guo, M. Sartor, M. Medvedovic, S. Kann, A. Puga, P. Ryan and C. R. Tomlinson (2004). Different global gene expression profiles in benzo[a]pyrene- and dioxin-treated vascular smooth muscle cells of AHR-knockout and wild-type mice. Cardiovasc Toxicol 4(1): 47-73.

Kurachi, M., S. Hashimoto, A. Obata, S. Nagai, T. Nagahata, H. Inadera, H. Sone, C. Tohyama, S. Kaneko, K. Kobayashi and K. Matsushima (2002). Identification of 2,3,7,8tetrachlorodibenzo-p-dioxin-responsive genes in mouse liver by serial analysis of gene expression. Biochem Biophys Res Commun 292(2): 368-77.

Lin, T. M., K. Ko, R. W. Moore, U. Simanainen, T. D. Oberley and R. E. Peterson (2002). Effects of aryl hydrocarbon receptor null mutation and in utero and lactational 2,3,7,8tetrachlorodibenzo-p-dioxin exposure on prostate and seminal vesicle development in C57BL/6 mice. Toxicol Sci 68(2): 479-87.

Martinez, J. M., C. A. Afshari, P. R. Bushel, A. Masuda, T. Takahashi and N. J. Walker (2002). Differential toxicogenomic responses to 2,3,7,8-tetrachlorodibenzo-p- dioxin in malignant and nonmalignant human airway epithelial cells. Toxicol Sci 69(2): 409-23.

Mootha, V. K., C. M. Lindgren, K. F. Eriksson, A. Subramanian, S. Sihag, J. Lehar, P. Puigserver, E. Carlsson, M. Ridderstrale, E. Laurila, N. Houstis, M. J. Daly, N. Patterson, J. P. Mesirov, T. R. Golub, P. Tamayo, B. Spiegelman, E. S. Lander, J. N. Hirschhorn, D. Altshuler and L. C. Groop (2003). PGC-1alpha-responsive genes involved in oxidative phosphorylation are coordinately downregulated in human diabetes. Nat Genet 34(3): 267-73.

Nohl, H., D. de Silva and K. H. Summer (1989). 2,3,7,8, tetrachlorodibenzo-p-dioxin induces oxygen activation associated with cell respiration. Free Radic Biol Med 6(4): 369-74.

Peters, J. M., M. G. Narotsky, G. Elizondo, P. M. Fernandez-Salguero, F. J. Gonzalez and B. D. Abbott (1999). Amelioration of TCDD-induced teratogenesis in aryl hydrocarbon receptor (AhR)-null mice. Toxicol Sci 47(1): 86-92.

Pfaffl, M. W. (2001). A new mathematical model for relative quantification in real-time RTPCR. Nucleic Acids Res 29(9): e45.

Puga, A., A. Maier and M. Medvedovic (2000). The transcriptional signature of dioxin in human hepatoma HepG2 cells. Biochem Pharmacol 60(8): 1129-42.

Ramakers, C., J. M. Ruijter, R. H. Deprez and A. F. Moorman (2003). Assumption-free analysis of quantitative real-time polymerase chain reaction (PCR) data. Neurosci Lett 339(1): 626. 
Schadt, E. E., C. Li, B. Ellis and W. H. Wong (2002). Extraction and Normalization Algorithm for High-density Oligonucleotide Gene Expression Array Data. Journal of Cellular Biochemistry 84(S37): 120-125.

Schlezinger, J. J., J. Keller, L. A. Verbrugge and J. J. Stegeman (2000). 3,3',4,4'-

Tetrachlorobiphenyl oxidation in fish, bird and reptile species: relationship to cytochrome P450 1A inactivation and reactive oxygen production. Comp Biochem Physiol C Toxicol Pharmacol 125(3): 273-86.

Schlezinger, J. J., R. D. White and J. J. Stegeman (1999). Oxidative inactivation of cytochrome P-450 1A (CYP1A) stimulated by 3,3',4,4'-tetrachlorobiphenyl: production of reactive oxygen by vertebrate CYP1As. Mol Pharmacol 56(3): 588-97.

Sedmera, D., T. Pexieder, M. Vuillemin, R. P. Thompson and R. H. Anderson (2000). Developmental patterning of the myocardium. Anat Rec 258: 319-337.

Seidman, J. G. and C. Seidman (2001). The genetic basis for cardiomyopathy: from mutation identification to mechanistic paradigms. Cell 104: 557-567.

Senft, A. P., T. P. Dalton, D. W. Nebert, M. B. Genter, R. J. Hutchinson and H. G. Shertzer (2002a). Dioxin increases reactive oxygen production in mouse liver mitochondria. Toxicol Appl Pharmacol 178(1): 15-21.

Senft, A. P., T. P. Dalton, D. W. Nebert, M. B. Genter, A. Puga, R. J. Hutchinson, J. K. Kerzee, S. Uno and H. G. Shertzer (2002b). Mitochondrial reactive oxygen production is dependent on the aromatic hydrocarbon receptor. Free Radic Biol Med 33(9): 1268-78.

Sorescu, D. and K. K. Griendling (2002). Reactive oxygen species, mitochondria, and NAD(P)H oxidases in the development and progression of heart failure. Congest Heart Fail 8(3): 132-140.

Staples, J. E., F. G. Murante, N. C. Fiore, T. A. Gasiewicz and A. E. Silverstone (1998). Thymic alterations induced by 2,3,7,8-tetrachlorodibenzo-p-dioxin are strictly dependent on aryl hydrocarbon receptor activation in hemopoietic cells. J Immunol 160(8): 3844-54.

Tang, Y. M., Y. Y. P. Wo, J. Stewart, A. L. Hawkins, C. A. Griffin, T. R. Sutter and W. F. Greenlee (1996). Isolation and characterization of the human cytochrome P450 CYP1B1 gene. J Biol Chem 271(45): 28324-30.

Teraoka, H., W. Dong, S. Ogawa, S. Tsukiyama, Y. Okuhara, M. Niiyama, N. Ueno, R. E. Peterson and T. Hiraga (2002). 2,3,7,8-Tetrachlorodibenzo-p-dioxin toxicity in the zebrafish embryo: altered regional blood flow and impaired lower jaw development. Toxicol Sci 65(2): 192-9.

Teraoka, H., W. Dong, Y. Tsujimoto, H. Iwasa, D. Endoh, N. Ueno, J. J. Stegeman, R. E. Peterson and T. Hiraga (2003). Induction of cytochrome P450 1A is required for 
circulation failure and edema by 2,3,7,8-tetrachlorodibenzo-p-dioxin in zebrafish. Biochem Biophys Res Commun 304(2): 223-8.

Toomey, B. H., S. Bello, M. E. Hahn, S. Cantrell, P. Wright, D. E. Tillitt and R. T. Di Giulio (2001). 2,3,7,8-Tetrachlorodibenzo-p-dioxin induces apoptotic cell death and cytochrome P4501A expression in developing Fundulus heteroclitus embryos. Aquat Toxicol 53(2): 127-38.

Walker, M. K. and T. F. Catron (2000). Characterization of cardiotoxicity induced by 2,3,7, 8tetrachlorodibenzo-p-dioxin and related chemicals during early chick embryo development. Toxicol Appl Pharmacol 167(3): 210-21.

Walker, N. J., C. J. Portier, S. F. Lax, F. G. Crofts, Y. Li, G. W. Lucier and T. R. Sutter (1999). Characterization of the dose-response of CYP1B1, CYP1A1, and CYP1A2 in the liver of female Sprague-Dawley rats following chronic exposure to 2,3,7,8-tetrachlorodibenzo-pdioxin. Toxicol Appl Pharmacol 154(3): 279-86.

Wisk, J. D. and K. R. Cooper (1990). The stage specific toxicity of 2,3,7,8-tetrachlorodibenzo-pdioxin in embryos of the Japanese medaka (Oryzias latipes). Environ Toxicol Chem 9: 1159-1169.

Yamazaki, K., H. Teraoka, W. Dong, J. J. Stegeman and T. Hiraga (2002). cDNA cloning and expressions of cytochrome P450 1A in zebrafish embryos. J Vet Med Sci 64(9): 829-33.

Zeytun, A., R. J. McKallip, M. Fisher, I. Camacho, M. Nagarkatti and P. S. Nagarkatti (2002). Analysis of 2,3,7,8-tetrachlorodibenzo-p-dioxin-induced gene expression profile in vivo using pathway-specific cDNA arrays. Toxicology 178(3): 241-60. 


\section{FIGURE LEGENDS}

Figure 1. Scatter plot of unadjusted median $\mathrm{Cy} 3$ and $\mathrm{Cy} 5$ feature intensities on a representative homotypic hybridization using zebrafish adult heart total RNA.

Figure 2. Dose-response curves for TCDD-induced pericardial edema (a) and peripheral circulatory impairment (b) in $80 \mathrm{hpf}$ embryos. Frequency (lines) of symptoms was determined from 100 randomly sampled embryos. Mean severity scores (bars) were calculated from ranks assigned to individual embryos according to the following scheme: $0=$ no adverse affect; $1=$ mild edema, or slowed caudal blood flow; 2 = moderate edema, or reduced blood cell number and flow rate; and 3 = severe edema, or no visible peripheral circulation.

Figure 3. Real-time RT-PCR data for control genes. a: mean expression ratios and standard errors for $\beta$-actin (circles) and ARNT (squares), as determined from two biological and two technical replicates. b: CYP1A expression ratios from duplicate measurements of two biological replicates.

Figure 4. Mean expression ratios and standard errors for troponin isoforms (a) and mitochondrial genes (b), as determined from real-time RT-PCR data for two biological replicates and two technical replicates. ctnnt $2=$ cardiac troponin T2; ss-tnnt $=$ skeletal slow muscle troponin $\mathrm{T}$; $\mathrm{sf}$-tnnt $=$ skeletal fast muscle troponin $\mathrm{T} ; \mathrm{CCO} 1=$ cytochrome $\mathrm{C}$ oxidase subunit 1 ; cytb $=$ cytochrome $b ; \mathrm{ND} 2=\mathrm{NADH}$ dehydrogenase subunit 2 . 
Table 1. Microarray data for exogenous and endogenous control probes. A. thaliana mRNAs were added to experimental samples in equimolar amounts to produce 1:1 ratios. Biological controls were unmanipulated.

\begin{tabular}{|c|c|c|c|c|}
\hline \multirow[b]{2}{*}{ Gene Name } & \multicolumn{2}{|c|}{$0.5 \mathrm{nM}$ TCDD } & \multicolumn{2}{|c|}{$5.0 \mathrm{nM}$ TCDD } \\
\hline & Ratio & P-value & Ratio & P-value \\
\hline \multicolumn{5}{|l|}{ A. thaliana spiked controls } \\
\hline chlorophyll a/b-binding protein (CAB) & 1.18 & 0.377 & -1.07 & 0.466 \\
\hline RUBISCO activase (RCA) & -1.29 & 0.287 & -1.29 & 0.007 \\
\hline RUBISCO large subunit (rbcL) & 1.34 & 0.264 & -1.11 & 0.446 \\
\hline \multicolumn{5}{|l|}{ Biological negative controls } \\
\hline$\beta$-actin & -1.02 & 0.971 & -1.29 & 0.109 \\
\hline ARNT & 1.26 & 0.345 & 1.23 & 0.263 \\
\hline \multicolumn{5}{|l|}{ Biological positive controls } \\
\hline aryl hydrocarbon receptor 2 (AHR2) & 1.85 & 0.000 & 1.93 & 0.000 \\
\hline cytochrome P450 1A (CYP1A) & 44.02 & 0.000 & 62.78 & 0.000 \\
\hline
\end{tabular}


Table 2. Known genes whose expression is significantly modulated by TCDD. ND = not detected; $\mathrm{NC}=$ no significant change ( $\mathrm{p}$-value $>0.005)$.

\section{(B) SARCOMERE COMPONENTS}

cardiac alpha actin

cardiac troponin $\mathrm{T} 2$

cardiac troponin $\mathrm{T} 2$

cardiac myosin light chain 1

cardiac myosin light chain 2

myosin light chain, similar to atrial

cardiac beta-myosin heavy chain ventricular myosin heavy chain myosin heavy chain, similar to cardiac myosin heavy chain, similar to slow muscle

\section{(C) MITOCHONDRIAL ENERGY TRANSFER}

cytochrome b

cytochrome c oxidase subunit I cytochrome c oxidase subunit III

NADH dehydrogenase subunit 1 NADH dehydrogenase subunit 2 $\mathrm{NADH}$ dehydrogenase subunit 4/4L $\mathrm{NADH}$ dehydrogenase subunit 5 $\mathrm{NADH}$ dehydrogenase subunit 6

ATP synthase ATP synthase 6 $\mathrm{H}+$-transporting ATP synthase

Gene Name
(A) METABOLIC PROCESSES
Xenobiotic metabolism
aryl hydrocarbon receptor 2 (AHR2)
cytochrome P450 1A (CYP1A)
cytochrome P450 1B1 (CYP1B1)
glutathione S transferase $\pi$ (GST $)$
major vault protein
Fatty acid metabolism
liver-basic fatty acid binding protein
acyl-coenzyme A dehydrogenase
trifunctional enzyme $\beta$ subunit (TP- $\beta$ )
Steroid synthesis
20 -hydroxysteroid dehydrogenase
Pyrimidine metabolism
pyrimidine 5' nucleotidase, cytosolic II \\ pyrimidine 5' nucleotidase, cytosolic III \\ PROCESES \\ aryl hydrocarbon receptor $2(A H R 2)$}

.

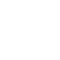

$0.5 \mathrm{nM}$ TCDD

\# Probes Ratio

P-value
$5.0 \mathrm{nM}$ TCDD Ratio P-value 
(D) RIBOSOMAL MACHINERY

mitochondrial 12s rRNA

mitochondrial 16s rRNA

60s ribosomal protein L39

ribosomal protein $\mathrm{L} 13 \mathrm{~A}$

40s ribosomal protein $\mathrm{S} 18$

60 s ribosomal protein 17 a

\begin{tabular}{|c|c|c|c|c|}
\hline 2 & -1.57 & 0.0002 & -1.76 & 0.0000 \\
\hline 1 & \multicolumn{2}{|c|}{-- NC -- } & -2.11 & 0.0000 \\
\hline 1 & \multicolumn{2}{|c|}{-- NC -- } & -1.35 & 0.000 \\
\hline 1 & \multicolumn{2}{|c|}{-- NC -- } & -1.16 & 0.00 \\
\hline 1 & -1.84 & 0.0035 & \multicolumn{2}{|c|}{-- NC -- } \\
\hline 1 & -1.92 & 0.0014 & \multicolumn{2}{|c|}{-- NC -- } \\
\hline
\end{tabular}

(E) MISCELLANEOUS

Ca2+ ATPase (SERCa)

Ca2+-transporting ATPase

small membrane protein 1

annexin Xla

protein kinase $\mathrm{c}$ inhibitor protein 1

cryptochrome 1a

beta-spectrin (sptb)

chaperonin containing t-complex protein 1

\begin{tabular}{lr}
1.55 & $-\mathrm{NC}-$ \\
1.29 & 0.0010 \\
1.40 & 0.0034 \\
1.50 & 0.0001 \\
1.81 & 0.0006 \\
1.83 & 0.0043 \\
1.94 & 0.0024 \\
\hline & 0.0000
\end{tabular}

$\begin{array}{ll}1.24 & 0.0040 \\ 1.40 & 0.0005 \\ 1.43 & 0.0000 \\ 1.40 & 0.0006 \\ 1.27 & 0.0015 \\ 1.85 & 0.0000 \\ & -- \text { NC -- } \\ & -- \text { NC -- }\end{array}$

Table 3. ESTs without known homologs and unidentified sequences whose expression is modulated by TCDD. $\mathrm{ND}=$ not detected; $\mathrm{NC}=$ no significant change $(\mathrm{p}$-value $>0.005)$.

\begin{tabular}{|c|c|c|c|c|c|}
\hline \multirow[b]{2}{*}{ Probe/Cluster ID } & \multirow[b]{2}{*}{ \# Probes } & \multicolumn{2}{|c|}{0.5 nM TCDD } & \multicolumn{2}{|c|}{5.0 nM TCDD } \\
\hline & & Ratio & P-value & Ratio & P-value \\
\hline \multicolumn{6}{|c|}{ (A) NO KNOWN HOMOLOGS } \\
\hline TR001 & 2 & 1.97 & 0.0000 & 2.33 & 0.0000 \\
\hline TR004 & 18 & 1.82 & 0.0000 & 2.12 & 0.0000 \\
\hline $\mathrm{AH} 045251$ & 1 & \multicolumn{2}{|c|}{-- ND -- } & 1.93 & 0.0002 \\
\hline $\mathrm{AH} 044370$ & 1 & \multicolumn{2}{|c|}{-- ND -- } & 2.05 & 0.0000 \\
\hline АH045914 & 1 & \multicolumn{2}{|c|}{-- NC -- } & 1.84 & 0.0001 \\
\hline $\mathrm{AH} 046765$ & 1 & \multicolumn{2}{|c|}{-- NC -- } & 1.83 & 0.0004 \\
\hline $\mathrm{AH} 045830$ & 1 & \multicolumn{2}{|c|}{-- NC -- } & 2.12 & 0.0000 \\
\hline $\mathrm{AH} 044267$ & 1 & \multicolumn{2}{|c|}{$--\mathrm{NC}--$} & 2.29 & 0.0002 \\
\hline $\mathrm{AH} 045770$ & 1 & 1.35 & 0.0016 & 1.17 & 0.0016 \\
\hline $\mathrm{AH} 046483$ & 1 & 1.38 & 0.0006 & 1.29 & 0.0014 \\
\hline $\mathrm{AH} 046317$ & 1 & 1.42 & 0.0006 & 1.53 & 0.0000 \\
\hline $\mathrm{AH} 046418$ & 1 & 1.44 & 0.0035 & 1.37 & 0.0001 \\
\hline $\mathrm{AH} 042619$ & 1 & 1.52 & 0.0001 & 2.21 & 0.0028 \\
\hline $\mathrm{AH} 045730$ & 1 & 1.75 & 0.0030 & 1.24 & 0.0001 \\
\hline $\mathrm{AH} 042622$ & 1 & 2.01 & 0.0017 & \multicolumn{2}{|c|}{-- ND -- } \\
\hline AH042585 & 1 & -1.45 & 0.0004 & -1.58 & 0.0002 \\
\hline $\mathrm{AH} 040736$ & 1 & -1.51 & 0.0000 & -1.22 & 0.0010 \\
\hline АН039190 & 1 & -1.84 & 0.0042 & -1.51 & 0.0719 \\
\hline uni2313 & 1 & -2.87 & 0.0036 & & C -- \\
\hline \multicolumn{6}{|c|}{ (B) LOW QUALITY SEQUENCE } \\
\hline $\mathrm{AH} 039140$ & 1 & \multicolumn{2}{|c|}{-- NC -- } & -2.19 & 0.0029 \\
\hline AH038856 & 1 & -1.76 & 0.0033 & -1.34 & 0.0050 \\
\hline AH042259 & 1 & -2.11 & 0.0059 & -1.86 & 0.0000 \\
\hline АН039112 & 1 & -1.96 & 0.0039 & \multicolumn{2}{|c|}{-- NC -- } \\
\hline
\end{tabular}




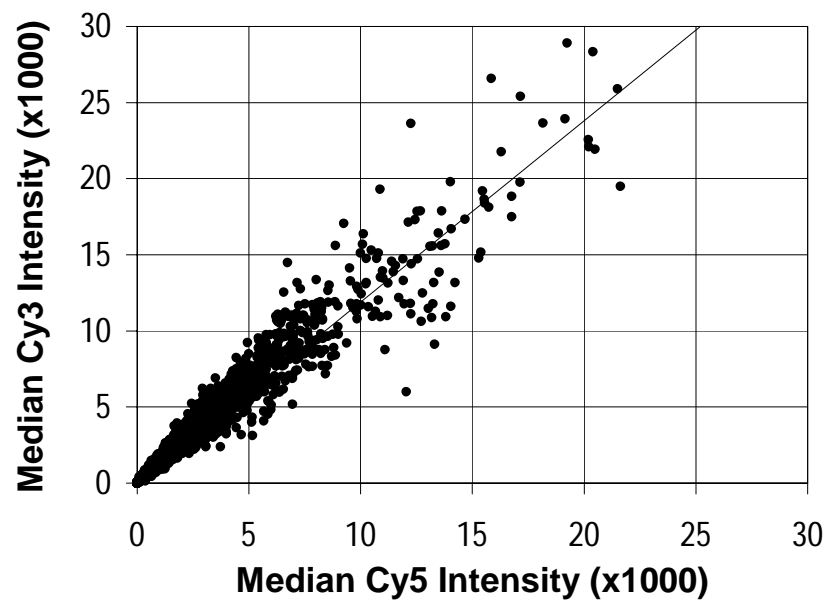


(a) pericardial edema

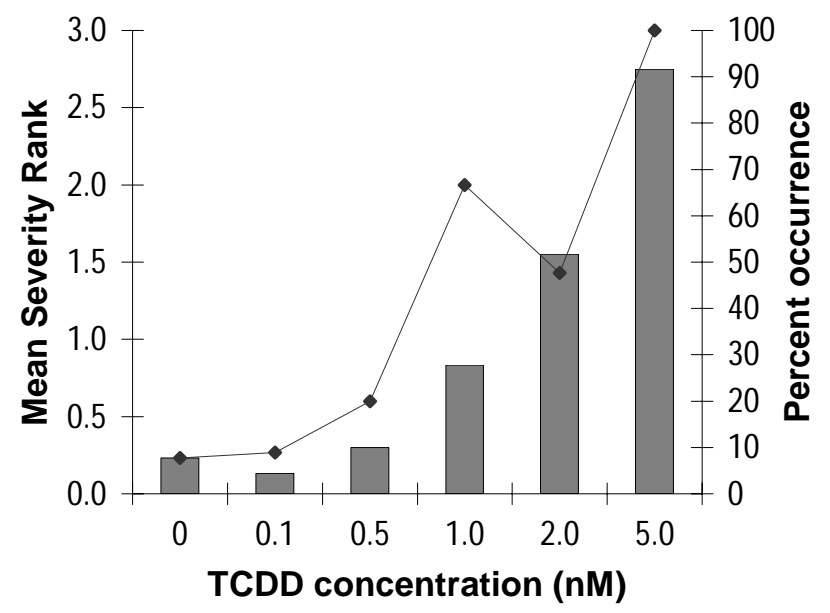

(b) circulatory impairment

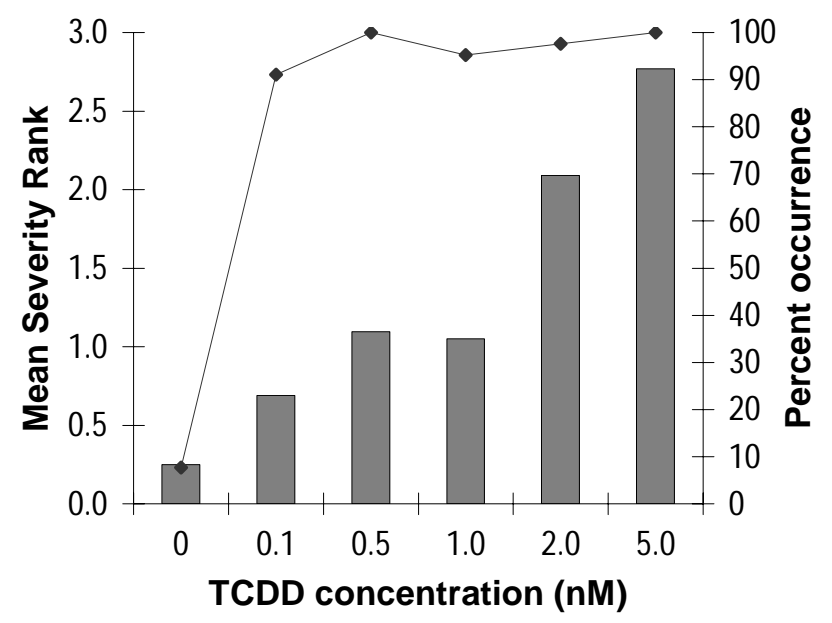


(a) $\beta$-actin and ARNT2

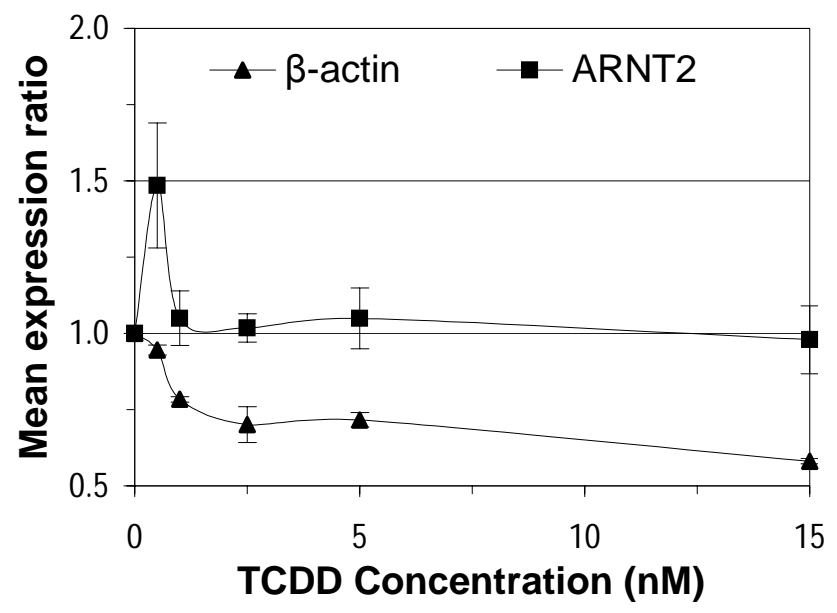

(b) CYP1A

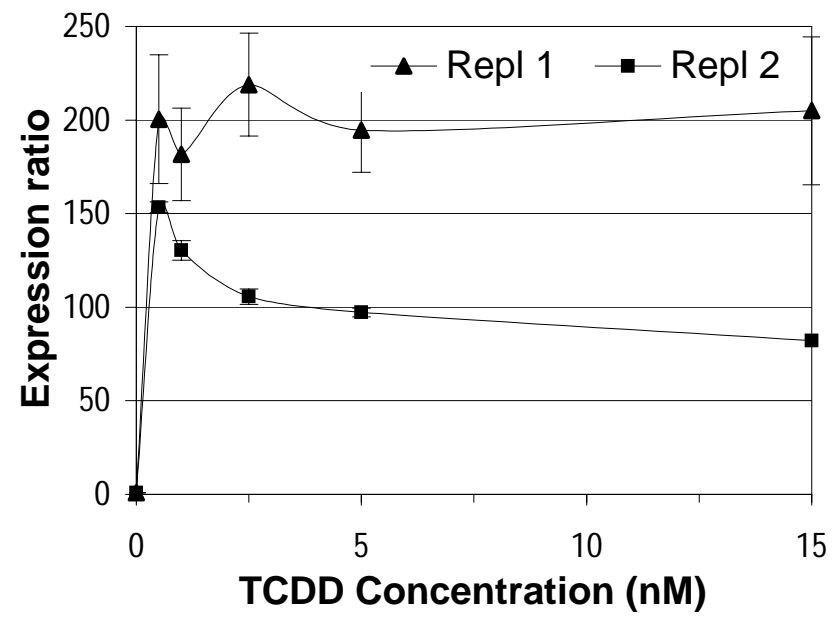


(a) troponin isoforms

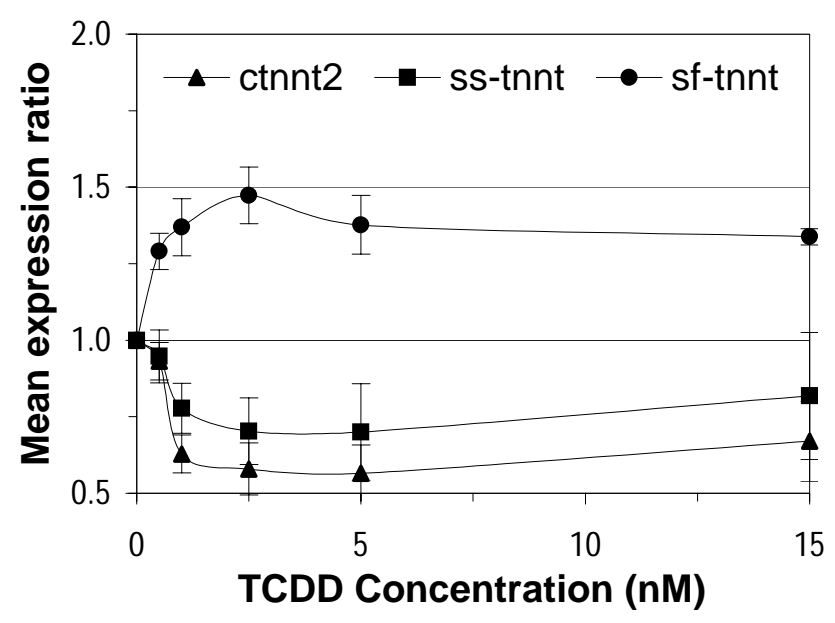

(b) mitochondrial genes

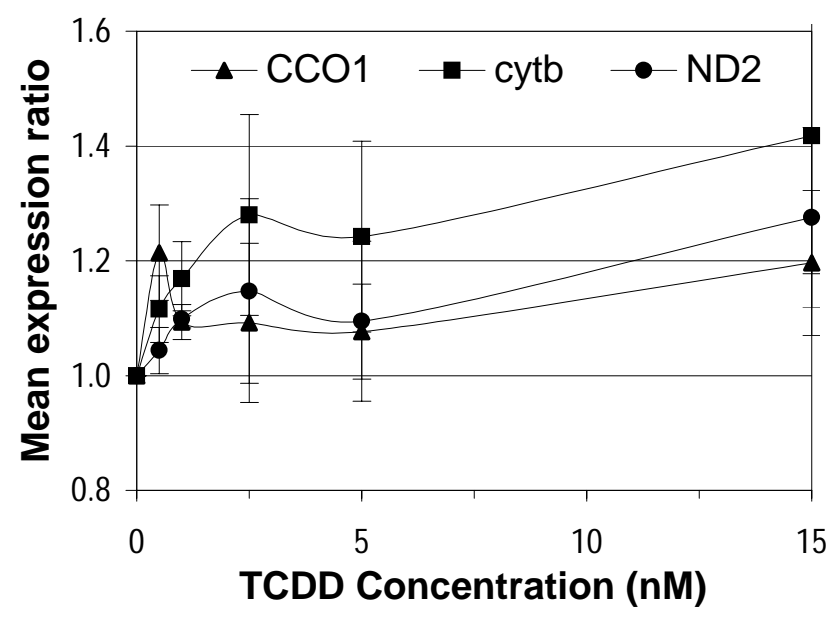

